\title{
„Die Branche schwankt zwischen vollkommen überlastet und beschäftigungslos"
}

\author{
Logistik und Mobilität seit Corona. Im Gespräch: Professor Tobias Bernecker, Hochschullehrer \\ für Verkehrswirtschaft und Verkehrspolitik an der Hochschule Heilbronn im Studiengang \\ Verkehrsbetriebswirtschaft und Logistik
}

\section{Vanessa Offermann ${ }^{1}$ Tobias Bernecker ${ }^{2}$}

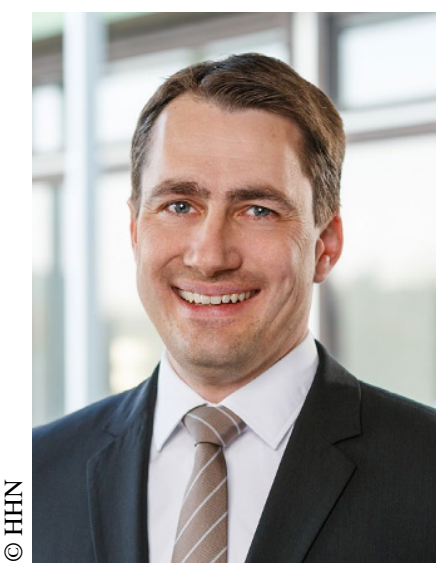

Professor Tobias Bernecker

In den ersten Corona-Wochen wurde die Bedeutung der Logistikbranche überdeutlich: Im Supermarkt war - für uns alle ungewohnt - der Nachschub an eigentlich selbstverständlichen Alltagsgütern wie Nudeln, Mehl und Toilettenpapier auf einmal nicht mehr gewährleistet. Obwohl sich in dieser Hinsicht Nachfrage und Angebot wieder eingependelt haben, bleiben Fragen eines (nachhaltigen) Transports von Menschen und Waren weiterhin Topthemen des 21. Jahrhunderts. Ende April, noch ganz unter dem Eindruck der starken Einschränkungen, sprach Professor Tobias Bernecker, Fachmann für die Bereiche Mobilität und Logistik

Vanessa Offermann

vanessa.offermann@hs-heilbronn.de

Tobias Bernecker

tobias.bernecker@hs-heilbronn.de

1 Hochschulkommunikation HS Heilbronn, Heilbronn, Deutschland

2 Verkehrsbetriebswirtschaft und Logistik, Kompetenzzentrum LOGWERT, Bildungscampus 9,74076 Heilbronn, Deutschland und zugleich Leiter des Kompetenzzentrums LOGWERT, einem Forschungszentrum für die Themenfelder Logistik und Verkehr, mit Vanessa Offermann, HS Heilbronn, Ende April 2020 über die aktuelle Situation dieser Branchen.

Wie ist denn derzeit, coronabedingt, die Situation in Mobilität und Logistik einzustufen?

Die Branche schwankt zwischen vollkommen überlastet und beschäftigungslos. Dort wo die Logistik unentbehrlich ist, um die Versorgung der Bevölkerung sicherzustellen, wird händeringend Personal gesucht. Viele Fahrer*innen sind am Limit. Andererseits mussten große Unternehmen wie die Lufthansa schon nach wenigen Tagen den operativen Betrieb - abgesehen vom Frachtgeschäft - nahezu einstellen, weil ihnen quasi über Nacht die Märkte weggebrochen sind.

Viele Ländergrenzen sind derzeit dicht. Wir sehen aber Paprika aus Spanien im Supermarkt. Wie funktioniert das?

Der Lkw darf fahren und auch die Grenzen passieren. Je nach Land sind aber unterschiedliche Zusatzerklärungen und Dokumente erforderlich, die viel Bürokratie verursachen. Im Internet kann man sich zum Beispiel live unter https://covid-19.sixfold.com/ informieren, wie lange es für Lkw derzeit dauert, um über eine bestimmte Grenze zu kommen. Auch die großen Containerschiffe auf den Weltmeeren sind nahezu wie gewohnt unterwegs. Dort beobachten wir in den letzten Tagen sogar einen Hauch von Normalität: Nachdem zu Beginn der Krise, ausgehend von China, die Frachtraten für Container weltweit zunächst stark gestiegen sind, normalisieren sie sich inzwischen schon wieder.

Lieferanten fahren beispielsweise quer durch Süditalien. Die Fahrt dauert mehrere Tage. Dabei wird ständig an 
Raststätten Halt gemacht. Können Sie Einblick geben, wie so eine Fahrt zur Corona-Zeit abläuft?

Sie ist in jedem Fall mit großem Stress für die Fahrer verbunden. Für den Güterkraftverkehr gelten Corona-Sonderregelungen dort, wo Waren des täglichen Bedarfs, medizinische Produkte und Treibstoffe zu befördern sind. Diese Lkw sind derzeit auch am Sonntag unterwegs, und die Fahrer dürfen ausnahmsweise regelmäßig bis zu zehn Stunden am Tag fahren, sofern sie sich fit fühlen. Pausen müssen natürlich dennoch gemacht werden, auch wenn das in der Praxis gar nicht so einfach ist: Auch auf den Rasthöfen gelten strenge Regelungen zu Kontaktverboten und umfassende HygieneVorschriften. Oder sie sind einfach geschlossen. Auf langen Touren ist das ein echtes Problem.

\section{Der öffentliche Verkehr wird derzeit eher gemieden. Wie} steht es um diese Branche?

Viele Städte und Gemeinden haben auch nach dem Lockdown Mitte März ihre Angebote im ÖPNV weitgehend aufrechterhalten, obwohl die Fahrgäste ausgeblieben sind. Die Nachfrage ist teilweise um bis zu $80 \%$ eingebrochen. Auch Bustouristik und Fernbusverkehr finden faktisch nicht mehr statt. Beides ist für die Branche wirtschaftlich nicht lange tragfähig. Ein Großteil der mittelständischen Buswirtschaft in Deutschland wird nach Schätzungen der Branchenverbände höchstens wenige Wochen durchhalten, wenn die Unternehmen nicht rasch und unbürokratisch finanzielle Hilfen erhalten.

\section{Werden die angekündigten Hilfspakete ausreichen?}

Das ist nicht sicher. Staatshilfen sollen ja eigentlich eine Ausnahme sein. Gerade der Busverkehr lebt vom Wettbewerb. Springt der Staat jetzt unüberlegt an zu vielen Stellen ein, laufen wir Gefahr, in wenigen Wochen einen funktionierenden Markt langfristig zu schädigen, der in Jahrzehnten aufgebaut worden ist. Und dies zu einem Zeitpunkt, zu dem es eigentlich für den öffentlichen Verkehr sehr gut gelaufen ist und immer mehr Menschen bereit waren, umzusteigen.

Stichwort Luftfahrt: Was machen die Flughäfen, wenn die Fluggäste ausbleiben?

Entweder Landebahnen als Abstellflächen an die Airlines vermieten, die dort ihre derzeit nicht benötigten Flugzeuge abstellen oder die Zeit nutzen, um Sanierungen vorzuziehen. Das ist beispielsweise in Stuttgart der Fall. Dort wurde die Krise als Chance genutzt, um in kürzester Zeit bei eingestelltem Betrieb einen Teil der Start- und Landebahn komplett zu sanieren. Ursprünglich hätte dies im Frühsommer unter laufendem Betrieb erfolgen sollen und wäre dann wesentlich komplizierter geworden.
Das Licht am Ende des Tunnels: Wie fährt man die Branche wieder hoch?

Nehmen wir die Automobillogistik als Beispiel: Rund $80 \%$ der Teile, Komponenten und Systeme eines Autos stammen von Zulieferern. Die dahinterstehende Logistik ist äußert komplex. Das kann nicht an einem Tag wieder hochgefahren werden. Ein Rad muss in das andere greifen und vieles muss beim Hochfahren gleichzeitig und nicht nacheinander geschehen. Nicht ohne Grund arbeiten Fahrzeughersteller und Logistiker schon seit Wochen Hand in Hand an konkreten Plänen, wie das ablaufen soll.

RED

Hinweis des Verlags Der Verlag bleibt in Hinblick auf geografische Zuordnungen und Gebietsbezeichnungen in veröffentlichten Karten und Institutsadressen neutral. 